Journal of Computer Science 7 (12): 1805-1812, 2011

ISSN 1549-3636

C 2011 Science Publications

\title{
Ergodic Capacity Analysis of Multiple Input Multiple Output Systems with Dual Hop Amplify and Forward Relay Networks
}

\author{
${ }^{1}$ C. Poongodi and ${ }^{2} \mathrm{~A}$. Shanmugam \\ ${ }^{1}$ Department of ECE, Bannari Amman Institute of Technology, \\ Sathyamagalam 638401, India
}

\begin{abstract}
Problem Statement: In MIMO system, capacity mainly depends on the channel and the antenna characteristics. The capacity can be improved by proper design of antenna elements and choosing appropriate array configuration and the directive elements give more capacity than omni directional elements. The dual polarized antennas are better than single polarized antennas as the former can provide two information channels. The mutual coupling also changes the capacity considerably. When the spacing between the elements is less than $0.5 \lambda$, the effect of mutual coupling is more. Approach: This study analyzes the capacity of Echelon, H-shaped; V-shaped free standing dipoles and printed dipole arrays of a MIMO system. The channel model for multi-polarized antennas is used in the simulations. The mutual coupling between the dipoles of different orientations is also included to make simulation more realistic. MIMO system with relay network is used to improve the coverage and reliability and to reduce the interference in wireless network. So we have analyzed the characterization of the ergodic capacity of amplify-and-forward (AF) MIMO dual-hop relay channels, assuming that the channel state information is available at the destination terminal only. Results: This study presents the results for channel capacity for different antenna configuration and the exact Ergodic capacity, closed form expression for the high SNR regime and tight closed form upper and lower bounds for amplify and forward relay system using the probability density function of an unordered eigenvalues. Conclusion/Recommendations: The Echelon, H-shaped; V-shaped free standing dipoles offer better capacity results in conventional MIMO system. The results produced are valid for all SNR values and for arbitrary numbers of antennas at the source, relay and destination. We also demonstrate a relationship between the conventional point-to-point MIMO Channels and dual-hop AF MIMO relay channel.
\end{abstract}

Key Words: Dipole configurations, ergodic capacity, relay network, amplify-and-forward, one ring Model, Decode-and-Forward (DF), Echelon configurations, conventional point

\section{INTRODUCTION}

In the last few years wireless services have become more and more important. Likewise the demand for higher network capacity and performance has been increased. In most wireless channels, no direct LOS propagation exists between the transmitter antennas and receive antenna because of natural and constructed obstacles. The transmitter signal may arrive at the receiver over many paths. This causes multi-path fading at a specific location and strength of the wave changes randomly. This multipath is often detrimental in case of single transmit and receive antennas. This unwanted is instead exploited in MIMO case to increase the channel capacity and quality (Foschini and Gans, 1998). In MIMO, both transmitter and receiver are provided with more than one antenna. In mobile communication environment, the wireless channel is time varying due to the mobility of the wireless terminal and multi-path propagation. MIMO performs well in scattering rich environment (Gesbert, 2003) . For rich scattering environment channel it is possible to increase the data rate by transmitting separate information streams on each antenna. In MIMO system, the capacity depends mainly on the channel and the antenna characteristics. The capacity can be improved by proper design of antenna elements (Svantesson and Ranheim, 2001) and choosing appropriate array configuration (Balanis, 1997) . Therefore it is important to know how various array configurations are performing in the case of MIMO system. The mutual coupling also changes the capacity considerably. When the spacing between the elements is less than $0.5 \lambda$, the effect of mutual coupling is more. 
In Dual hop relay network there are three main types of relaying protocols: Decode-and-Forward (DF), Compress-and-Forward (CF) and Amplify-and-Forward $(\mathrm{AF})$. Of these protocols, the AF approach is the simplest scheme, in which case the sources transmit messages to the relays, which then simply scale their received signals according to a power constraint and forward the scaled signals onto the destinations. The Ergodic mutual information of the i.i.d. Rayleigh fading amplify-and-forward MIMO relay channel without direct link between source and destination is investigated in (Wang et al., 2009). By means of the replica method an expression is obtained, whose parameters are determined by a nonlinear equation system. This system can be solved in closed form and also shows comparisons with Monte Carlo simulations that the asymptotic expression serves as an excellent approximation. They analysed fading interference relay networks where $M$ single-antenna source-destination terminal pairs communicate concurrently and in the same frequency band through a set of K single-antenna relays using half-duplex two-hop relaying. The relays do not have channel state information, perform Amplify-and-Forward (AF) relaying and the destination terminals can cooperate and perform joint decoding.

An asymptotic Ergodic capacity of MIMO Amplify-and-Forward (AF) relaying systems which employ linear processing at the relay is analysed in (Lee et al., 2010). By exploiting the asymptotic results for Eigen value distributions and also derived the Ergodic capacity in various asymptotic antenna regimes as a closed-form expression with arbitrary system parameters. The analyzed results demonstrate that increasing the number of source antennas causes the capacity shrink phenomenon which is analogous to the channel hardening effect in multi-user MIMO systems. Lee et al. (2010) the author assumed asymptotically large antennas to obtain the closed-form expressions, simulation results show that his derived expressions are surprisingly accurate even with the moderate number of antennas and thus can serve for analyzing practical MIMO relay networks.

Fading interference relay networks where $\mathrm{M}$ single-antenna source-destination terminal pairs communicate concurrently and in the same frequency band through a set of $\mathrm{K}$ single-antenna relays using half-duplex two-hop relaying is analysed (Morgenshtern and Bolcskei, 2006). The relays do not have channel state information, perform amplify-andforward (AF) relaying and the destination terminals can cooperate and perform joint decoding.

\section{MATEIALS AND METHODS}

MIMO system capacity and channel model: For a narrow band MIMO channel, when CSI is not known at the transmitter, the capacity is given by (Paulraj et al., 2003):

$$
\mathrm{C}=\mathrm{E}\left[\log _{2}\left(\operatorname{det}\left(\mathrm{I}_{\mathrm{Nr}}+\mathrm{pHH}^{+} / \mathrm{N}\right)\right)\right]
$$

Where:

$\rho=$ The average signal to noise ratio at each receiver

$\mathrm{H}=$ The $\mathrm{N}_{\mathrm{r}} \times \mathrm{N}_{\mathrm{t}}$ channel matrix

$\mathrm{N}=\min \left(\mathrm{N}_{\mathrm{t}}, \mathrm{N}_{\mathrm{r}}\right) \cdot \mathrm{N}_{\mathrm{t}}$

$\mathrm{N}_{\mathrm{r}}=$ The number of transmitting and receiving antennas respectively

More often each element of $\mathrm{H}$ is taken to be i.i.d complex gaussian distributed random variable signifying that each pair of transmit and receive antennas experiences independent fading. But, this is not true in practical situation. Because of spacing and mutual coupling between the elements, independent fading is not a valid assumption. In (Svantesson and Ranheim, 2001) the effect of coupling between antenna elements is included in the channel matrix as given below:

$$
\mathrm{H}_{\mathrm{mc}}=\mathrm{C}_{\mathrm{b}} \mathrm{HC}_{\mathrm{m}}
$$

Where:

$\mathrm{C}_{\mathrm{b}}=$ The coupling matrix at the base station

$\mathrm{Cm}=$ Coupling matrix at the mobile. Now the capacity is modified as:

$$
\mathrm{C}=\mathrm{E}\left[\log _{2}\left(\operatorname{det}\left(\mathrm{I}_{\mathrm{Nr}}+\mathrm{pH}_{\mathrm{mc}} \mathrm{h}_{\mathrm{mc}}{ }^{+} / \mathrm{N}\right)\right)\right]
$$

The expression for coupling matrix is defined as (Svantesson and Ranheim, 2001):

$$
\mathrm{C}=\left(\mathrm{Z}_{\mathrm{A}}+\mathrm{Z}_{\mathrm{T}}\right)\left(\mathrm{Z}+\mathrm{Z}_{\mathrm{T}} \mathrm{l}\right)^{1}
$$

$\mathrm{Z}_{\mathrm{A}}=$ Antenna impedance in isolation

$\mathrm{I}=$ Identity matrix

$\mathrm{Z}_{\mathrm{T}}=$ The impedance of the receiver at each antenna element, chosen as the complex conjugate

$\mathrm{Z}_{\mathrm{A}}=$ To obtain an impedance match for maximum power transfer

$\mathrm{Z}=$ The mutual impedance matrix.

To include the channel characteristics, the one ring channel model proposed in (Svantesson, 2001) is used here in the simulation. It is a widely used model for microcell and picocell environment (Poongodi et al., 2010). 


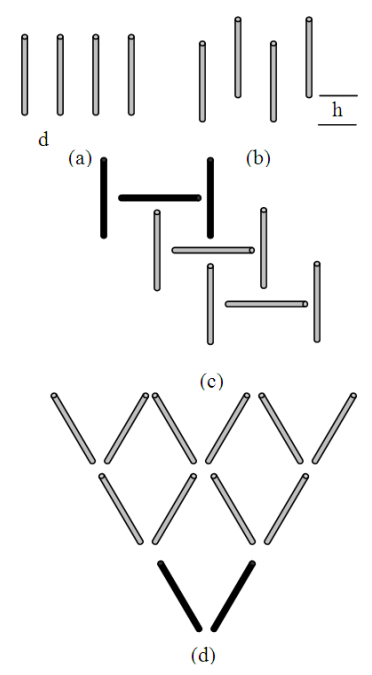

Fig. 1: (a) Side by Side (b) Echelon (c) H shaped (d) $\mathrm{V}$ shaped dipole array configurations

Antenna configurations: The antenna array configurations investigated in this study are: Side by side, Echelon, $\mathrm{H}$ shaped and V shaped arrays. They are shown in Fig. 1.

As the side by side and echelon configurations have their elements oriented in the vertical direction, they provide only space diversity (Carter, 1932) unlike $\mathrm{H}$ and $\mathrm{V}$ shaped arrays. The $\mathrm{H}$ and $\mathrm{V}$ shaped free standing dipole arrays are dual polarized. They can give more capacity. In a finite array of printed dipoles, each dipole is assumed to have a length $\mathrm{L}$, a width $\mathrm{W}$ and to be uniformly spaced from its neighbors by distance ' $a$ ' in the $\mathrm{x}$-direction and ' $\mathrm{b}$ ' in the $\mathrm{y}$ direction. The poles are assumed to be thin $(\mathrm{W}<<\mathrm{L})$ so only $\mathrm{x}$ directed currents are required. The green's function representing $\mathrm{E}_{\mathrm{x}}(\mathrm{x}, \mathrm{y})$ due to an infinitesimal electric dipole of unit strength lying along the $\mathrm{x}$-direction on the dielectric slab at $\left(\mathrm{x}_{0}, \mathrm{y}_{0}\right)$ can be expressed as:

$$
E_{x}(x, y)=\frac{-j Z_{0}}{4 \pi^{2} k_{0}} \int_{-\infty}^{\infty} \int Q\left(k_{x}, k_{y}\right) e^{j k_{x}\left(x-x_{0}\right)} e^{j k_{y}\left(y-y_{0}\right)} \mathrm{dk}_{x} d k_{y}
$$

The electric surface current density on the dipoles is expanded in terms of Piece Wise Sinusoidal (PWS) modes, defined as:

$$
\mathrm{J}_{\mathrm{i}}\left(\mathrm{x}_{0}, \mathrm{y}_{0}\right)=\frac{\operatorname{sink}_{\mathrm{e}}\left(\mathrm{h}-\left|\mathrm{x}_{0}-\mathrm{x}_{\mathrm{i}}\right|\right)}{\mathrm{W} \operatorname{sink} \mathrm{e}}
$$

For $\mathrm{X}_{0}-\mathrm{X}_{\mathrm{i}}<\mathrm{h}, \mathrm{h}$ is the half length of the expansion mode and $\mathrm{K}_{\mathrm{e}}$ is the wave number of the expansion mode, where:

$$
\mathrm{K}_{\mathrm{e}}=\mathrm{K}_{0} \sqrt{\left(\varepsilon_{\mathrm{r}}+1\right) / 2}
$$

An electric field integral eq can be solved using a Galerkin moment method solution (Pozar, 1985; Pozar, 1982).

Dipole pattern expressions: The free standing dipoles are oriented in different directions in the above shown configurations. To evaluate the channel fading we need the radiation pattern expressions $G_{t}$ and $G_{r}$. The normalized electric field pattern for $\overrightarrow{\mathrm{z}}$ directed $\lambda / 2$ dipole is given by (Svantesson and Ranheim, 2001):

$\mathrm{G} \theta=\frac{\cos \left[\frac{\pi}{2} \cos \theta\right]}{\sin \theta}$

The orientation of the transmitted field is given by:

$\mathrm{g}_{\mathrm{t}}=\mathrm{a}_{\varnothing}=\mathrm{a}_{\mathrm{x}} \operatorname{coa} \varnothing \cos \varnothing+\mathrm{a}_{\mathrm{y}} \cos \varnothing \sin \varnothing-\mathrm{a}_{\mathrm{z}} \sin \emptyset(8 \mathrm{a})$

For the simulation of $\mathrm{H}$ shaped structures, the pattern expressions for horizontal ' $y$ ' directed dipole must be found and is given by:

$$
\begin{gathered}
\mathrm{G} \theta=\frac{\cos \left[\frac{\pi}{2} \sin \theta \sin \phi\right]}{1-(\sin \theta \sin \phi)^{2}} \cos \theta \sin \phi \\
\mathrm{G} \phi=\frac{\cos \left[\frac{\pi}{2} \sin \theta \sin \phi\right]}{1-(\sin \theta \sin \phi)^{2}} \cos \theta \sin \phi
\end{gathered}
$$

The orientation of the field for this case is determined by the values of components along $\mathrm{a}_{\varnothing}$ and $a_{\varnothing}$. For the simulation of $\mathrm{V}$ shaped antennas, the pattern of the slanted dipoles are required. It can be easily found out by the vector potential due to slanted dipole into horizontal and vertical components and proceeding in the usual way of finding the field expressions:

$$
\begin{aligned}
& \cos \psi=\sin \theta \cos \phi \cos \gamma+\sin \theta \sin \phi \sin \gamma \\
& \mathrm{A}_{\mathrm{y}}=\cos \mathrm{y} \frac{\cos \left[\frac{\pi}{2} \cos \psi\right]}{\sin ^{2} \psi} \& \mathrm{~A}_{\mathrm{z}}=\sin \gamma \frac{\cos \left(\frac{\pi}{2} \cos \psi\right)}{\sin \psi} \\
& \mathrm{G}_{\theta}=\cos \theta \sin \phi \mathrm{A}_{\mathrm{y}}-\sin \theta \mathrm{A}_{\mathrm{z}} \\
& \mathrm{G}_{\phi}=\cos \phi \mathrm{A}_{\mathrm{y}}
\end{aligned}
$$

Here $\gamma$ is the slanting angle. For the printed dipole antenna the radiation pattern and the orientation pattern of the transmitted and received fields are defined in (Garg et al., 2001). 
Mutual coupling expression for free standing and printed dipole antenna: The mutual impedance for side by side and Echelon configurations is defined in (Balanis, 1997). For V shaped antenna mutual coupling calculation the mutual impedance between two slanted dipoles is used and it is given by (Carter, 1932):

$$
\mathrm{Z}_{21}=30 \mathrm{I}\left[\int_{0}^{1} \frac{\operatorname{sinkr}}{\mathrm{r}_{2}} \frac{\operatorname{sinks}}{\mathrm{s}} \mathrm{ds}+\mathrm{j} \int_{0}^{1} \frac{\operatorname{coskr} \mathrm{r}_{2}}{\mathrm{r}_{2}} \frac{\operatorname{sinks}}{\mathrm{s}} \mathrm{ds}\right]
$$

where, $r_{2} \sqrt{1^{2}+s^{2}+21 s \cos 2 \gamma}$ and $1=0.5 \lambda$. The mutual impedance between two slanted dipoles are computed numerically and used in the simulations.

Method Of Moment (MOM) solution is used to find the mutual impedance of the printed dipole array (Pozar, 1983). The impedance matrix of the array is given by:

$$
\mathrm{Z}_{\mathrm{mn}}=\frac{-\mathrm{j} \mathrm{Z}_{0}}{4 \pi^{2} \mathrm{k}_{0}} \int_{-\infty}^{\infty} \int \mathrm{Q}\left(\mathrm{k}_{\mathrm{x}}, \mathrm{k}_{\mathrm{y}}\right) \mathrm{F}_{\mathrm{m}}\left(\mathrm{k}_{\mathrm{x}}, \mathrm{k}_{\mathrm{y}}\right) \mathrm{F}_{\mathrm{n}}^{*}\left(\mathrm{k}_{\mathrm{x}}, \mathrm{k}_{\mathrm{y}}\right) \mathrm{dk}_{\mathrm{x}} \mathrm{dk_{ \textrm {y } }}
$$

Where:

Fn $=$ The Fourier transform of the expansion mode on the dipole $n$ :

$$
F_{n}\left(k_{x}, k_{y}\right)=\int_{x_{n}-h}^{x_{n}+h} \int_{y_{n}-w / 2}^{y_{n}+w / 2} J_{n}(x, y) e^{j k_{x} x} e^{j k_{y} y} d x d y
$$

The double infinite integration can be reduced to a finite integration and a semi-infinite integration by converting to polar coordinates $(\beta, \alpha)$ in the spectral domain. In addition convergence can be accelerated by using the techniques of (Pozar, 1983), whereby a term representing the contribution of the current in a homogeneous medium is subtracted from the green's function of the dielectric slab. So the Eq. 13 can then be written as:

$$
\begin{aligned}
& Z_{\mathrm{mn}}=\frac{\mathrm{j} \mathrm{Z}_{\mathrm{o}}}{4 \pi^{2} \mathrm{k}_{0}} \int_{\mathrm{B}-0}^{\infty} \int_{\mathrm{c}-0}^{2 \mathrm{x}} \mathrm{Q}^{\mathrm{h}} \mathrm{F}_{\mathrm{m}} \mathrm{F}_{\mathrm{n}} \mathrm{BdBda} \\
& +\frac{\mathrm{jZ} 0}{4 \pi^{2} \mathrm{~K} 0} \int_{\beta=0}^{\infty} \int_{\alpha=0}^{2 \pi}\left[\mathrm{Q}-\mathrm{Q}^{\mathrm{h}}\right] \mathrm{F}_{\mathrm{m}} \mathrm{F}_{\mathrm{n}} \beta d \beta d \alpha
\end{aligned}
$$

where, $Q^{\text {h }}$ represents the source in an effective homogeneous medium and is defined in (Pozar, 1983).

Dual-hop AF MIMO system: In this study we analysed the Ergodic capacity for simple closed-form high SNR and tight closed-form upper and lower bounds AF MIMO dual-hop systems. The results are based heavily on the theory of finite-dimensional random matrices. Based on analytical expressions, we investigate the effect of the different system and channel parameters on the Ergodic capacity. By adding more antennas at the destination, while keeping the number of source and destination antennas fixed, may lead to a significant improvement in the high SNR power offset; however the relative gain becomes less significant as the initial number of destination antennas is increased.

System model: We employ the same AF MIMO dualhop system model as in (Wagner et al., 2007; Morgenshtern and Bolcskei, 2006). In particular, suppose that there are $\mathrm{n}_{\mathrm{s}}$ source antennas, $\mathrm{n}_{\mathrm{r}}$ relay antennas and $n_{d}$ destination antennas, which we represent by the 3-tuple $\left(n_{s}, n_{r}, n_{d}\right)$. All terminals operate in half-duplex mode and as such communication occurs from source to relay and from relay to destination in two separate time slots. It is assumed that there is no direct communication link between the source and destination. The end-to-end input-output relation of this channel is then given by:

$$
\mathrm{y}=\mathrm{H}_{2} \mathrm{FH} \mathrm{s}+\mathrm{H}_{2} \mathrm{Fn}_{\mathrm{n}_{\mathrm{r}}}+\mathrm{n}_{\mathrm{n}_{\mathrm{d}}}
$$

Where: $\mathrm{s}$ is the transmit symbol vector, $\mathrm{n}_{\mathrm{nr}}$ and $\mathrm{n}_{\mathrm{nd}}$ are the relay and destination noise vectors respectively, $\mathrm{F}=\sqrt{\alpha /\left(\mathrm{n}_{\mathrm{r}}(1+\rho)\right)} \mathrm{I}_{\mathrm{n}_{\mathrm{r}}} \quad(\alpha$ corresponds to the overall power gain of the relay terminal) is the forwarding matrix at the relay terminal which simply forwards scaled versions of its received signals and $\mathrm{H} 1 \in \mathrm{C}{ }^{\mathrm{nr}} \times \mathrm{ns}$ and $\mathrm{H} 2 \in \mathrm{C}^{\text {ndxnr }}$ denote the channel matrices of the first hop and the second hop respectively, where their entries are assumed to be zero mean circular symmetric complex Gaussian (ZMCSCG) random variables of unit variance. The input symbols are chosen to be independent and identically distributed (i.i.d.) ZMCSCGs and the per antenna power is assumed to be $\rho / n s$, i.e., $E\left\{s^{*}\right\}=\left(p / n_{s}\right) 1_{n s}$. The additive noise at the relay and destination are assumed to be white in both space and time and are modeled as ZMCSCG with unit variance, i.e., $\operatorname{Eb}\left\{\mathrm{n}_{\mathrm{nr}} * \mathrm{n}_{\mathrm{nr}} *\right\}=1_{\mathrm{nr}}$ and $\operatorname{Eb}\left\{\mathrm{n}_{\mathrm{nd}} * \mathrm{n}_{\mathrm{nd}} *\right\}=1_{\mathrm{nd}}$. We assume that the source and relay have no Channel State Information (CSI) and that the destination has perfect knowledge of both $\mathrm{H} 2$ and $\mathrm{H} 1$. The Ergodic capacity (in $\mathrm{b} / \mathrm{s} / \mathrm{Hz}$ ) of the AF MIMO dual-hop system described above can be written as (Wagner et al., 2007; Morgenshtern and Bolcskei, 2006; Morgenshtern and Bolcskei, 2007; Sayadi et al., 2009):

$$
\mathrm{C}=\frac{1}{2} \mathrm{E}\left\{\log _{2} \operatorname{det}\left(\mathrm{I}+\mathrm{R}_{\mathrm{s}} \mathrm{R}_{\mathrm{n}}^{-1}\right)\right\}
$$

where, $R_{s}$ and $R_{n}$ are $n_{d} \times n_{d}$ matrices given $\mathrm{b} \mathrm{R}_{\mathrm{s}}=\frac{\rho \mathrm{a}}{\mathrm{n}_{\mathrm{s}}} \mathrm{H}_{2} \mathrm{H}_{1} \mathrm{H}_{1}^{*} \mathrm{H}_{2}^{*}$ and $\mathrm{R}_{\mathrm{n}}=\mathrm{I}_{\mathrm{n}_{\mathrm{d}}}+\mathrm{aH}_{2} \mathrm{H}_{2}^{*}$ 
Eq. 16 can be alternatively expressed as follows:

$C(\rho)=\frac{1}{2} E\left\{\log _{2} \operatorname{det}\left(1_{n s}+\frac{\rho a}{n_{s}} H_{1}^{*} H_{2}^{*} R_{n}^{-1} H_{2} H_{1}\right)\right\}$

By using singular value decomposition the Ergodic capacity in (17) can be further simplified as:

$C(\rho)=\frac{1}{2} E\left\{\log _{2} \operatorname{det}\left(1_{n s}+\frac{\rho a}{n_{s}} H_{1}^{*} \phi H_{1}\right)\right\}$

$\psi=\left\{\begin{array}{l}\operatorname{diag} \frac{\chi_{1}^{2}}{1+a x_{1}^{2}}, \ldots ., \frac{x_{n r}^{2}}{1+a \chi_{n r}^{2}} \\ \operatorname{diag}\{\frac{\chi_{1}^{2}}{1+a \chi_{1}^{2}}, \ldots ., \frac{\chi_{n}^{2}}{1+a \chi_{n r}^{2}} \underbrace{0, \ldots, 0}_{n_{r}-n_{d}}\}\end{array}\right\} \begin{array}{ll}n_{\mathrm{r}} & \leq n_{d} \\ n_{\mathrm{r}} & >\mathrm{n}_{\mathrm{d}}\end{array}$

$\mathrm{L}=\operatorname{diag}\left\{\mathrm{y}_{\mathrm{j}}^{2} /\left(1+\mathrm{a} \mathrm{y}_{\mathrm{j}}^{2}\right)\right\}_{\mathrm{j}}^{\mathrm{q}}=1$

where $\mathrm{q}=\min \left(\mathrm{n}_{\mathrm{d}}, \mathrm{n}_{\mathrm{r}}\right)$

Equivalently, we can now write:

$C(\rho)=\frac{s}{2} \int_{0}^{\infty} \log _{2}\left(1+\frac{\rho \alpha}{n_{s}} \chi\right) f \chi(\chi) d \chi$

where, $\mathrm{s}=\min (\mathrm{ns}, \mathrm{q}), \lambda$ denotes an unordered eigenvalue of the random matrix $\mathrm{H}_{1} * \mathrm{LH}_{1}$ and $\mathrm{f} \lambda($.) denotes the corresponding probability density function (p.d.f.). Although the distribution of $\lambda$ has been wellstudied in the asymptotic antenna regime (Wagner et al., 2007; Morgenshtern and Bolcskei, 2006), currently there are no exact closed-form expressions for $\mathrm{f}(\cdot)$ which apply for arbitrary finite-antenna systems.

Ergodic capacity analysis: The analytical expressions for the Ergodic capacity of AF MIMO dual-hop system is given by:

$\mathrm{C}(\rho)=\mathrm{K} \sum_{1-1}^{\mathrm{q}} \sum_{\mathrm{k}-\mathrm{q}-\mathrm{s}+1}^{\mathrm{q}} \sum_{\mathrm{j}-0}^{\mathrm{q}+\mathrm{n}_{\mathrm{s}}-1} \frac{\left(\mathrm{q}+\mathrm{n}_{\mathrm{s}}^{-1}\right) \mathrm{a}_{\mathrm{q}+\mathrm{ns}}^{-1-\mathrm{s}}}{\Gamma\left(\mathrm{n}_{\mathrm{s}}^{-\mathrm{q}+\mathrm{k}}\right)} \mathrm{Gl}, \mathrm{kJ}_{\mathrm{i}, \mathrm{k}}$

Where:
$\mathrm{J}_{\mathrm{i}, \mathrm{k}}=\int_{0}^{\infty} \log _{2}\left[1+\frac{\rho \mathrm{a}}{\mathrm{n}_{\mathrm{s}}} \chi\right] \mathrm{e}-{ }^{\mathrm{x} \alpha} \chi_{\mathrm{n}}^{2}+{ }^{2 \mathrm{k}+\mathrm{p}-\mathrm{q}-\mathrm{j}-3} \mathrm{~K}_{\mathrm{p}+\mathrm{q}=\mathrm{j}-1}(2 \sqrt{\chi}) \mathrm{d} \chi$

The integral in $\mathrm{J}_{\mathrm{i}, \mathrm{k}}$ can be evaluated either numerically, or can be expressed as an infinite series involving Meijer-G functions.

High SNR capacity analysis: For the high SNR regime, we consider two important scenarios; namely, one where the source and relay powers grow large proportionately and one where the source power grows large but the relay power is kept fixed:

If Large Source Power, Large Relay Power, then we have $\alpha \rightarrow \infty, \rho \rightarrow \infty$, with $\alpha / \rho=\beta$, for some fixed $\beta$. Then $\rho \alpha \rightarrow \frac{\alpha}{\mathrm{n}_{\mathrm{r}}}$ and $\alpha / \beta / \mathrm{n}_{\mathrm{r}}$ and the Ergodic capacity at high SNR reduces to:

$$
\left.C(\rho)\right|_{\alpha, \rho \rightarrow \infty, \alpha / \rho=\beta}=\frac{1}{2} E\left\{\left(I_{n_{s}}+\frac{\rho \beta}{n_{s} n_{r}} \tilde{H}_{1}^{*} \bar{L}_{2} \tilde{H}_{1}\right)\right\}
$$

Here, the two key parameters are $\mathrm{S} \propto$, which denotes the high-SNR slope in bits/s/Hz/ (3 dB) given by:

$\mathrm{S}_{\infty}=\lim _{\alpha, \rho \rightarrow \infty}\left(\frac{\left.\mathrm{C}(\rho)\right|_{\alpha, \rho \rightarrow \infty, \alpha / \rho=\beta}}{\log _{2}(\rho)}\right)$

And L $\infty$, which represents the high-SNR power offset in $3 \mathrm{~dB}$ units given by:

$$
\mathrm{L}_{\infty}=\lim _{\alpha, \rho \rightarrow \infty}\left(\log _{2}(\rho)-\frac{\left.\mathrm{C}(\rho)\right|_{\alpha, \rho \rightarrow \infty, \alpha / \rho=\beta}}{\mathrm{S}_{\infty}}\right)
$$

Tight bounds on the ergodic capacity: In order to obtain further simplified closed-form results, the upper and lower bound on the Ergodic capacity is given. The Ergodic capacity of AF MIMO dual-hop systems is upper bounded by:

$\mathrm{C}(\rho) \leq \mathrm{C}_{\mathrm{U}}(\rho)=\frac{1}{2} \log _{2}(\kappa \operatorname{det}(\overline{\bar{\Xi}}))$

where, $\bar{\Xi}$ is defined as:

$\{\Xi\}_{\mathrm{m}, \mathrm{n}}=\left\{\begin{array}{c}\Gamma(\tau-1), \\ \Gamma(\tau-1)\left(1+\frac{\alpha}{n_{\mathrm{s}} \mathrm{n}_{\mathrm{r}}}\left(\mathrm{n}_{\mathrm{s}}-\mathrm{q}+\mathrm{n}\right)(\tau-1)\right), \begin{array}{l}\mathrm{n} \leq \mathrm{q}-\mathrm{n}_{\mathrm{s}} \\ \mathrm{n} \leq \mathrm{q}-\mathrm{n}_{\mathrm{s}}\end{array}\end{array}\right.$ 
With $\mathrm{T}=\mathrm{p}-\mathrm{q}+\mathrm{m}+\mathrm{n}$ The Ergodic capacity of AF MIMO dual-hop systems is lower bounded by:

$\mathrm{C}(\rho) \geq \mathrm{C}_{\mathrm{L}}(\rho)=\frac{\mathrm{s}}{2}\left(1+\frac{\rho \mathrm{a}}{\mathrm{n}_{\mathrm{s}}} \exp \left(\left[\sum_{\mathrm{s}}^{\mathrm{s}} \psi\left(\sum_{\mathrm{k}=\mathrm{q}}^{\mathrm{s}} \psi\left(\mathrm{n}_{\mathrm{s}}-\mathrm{s}+\mathrm{k}\right)+\mathrm{k}+1 \mathrm{det}\left(\mathrm{W}_{\mathrm{k}}\right)\right]\right)\right)\right.$

The lower bound is clearly seen to be tight for the entire range of SNRs. Moreover, in the high SNR regime (e.g., $\rho \approx 15 \mathrm{~dB}$ ), the lower bound and exact capacity curves coincide.

\section{RESULTS}

To have pico-cell case, the distance between BS and MS are assumed to be $100 \mathrm{~m}$. About 40 scatterers are uniformly distributed within a ring of radius $30 \mathrm{~m}$. The frequency of operation is $5 \mathrm{GHz}$. The SNR is varied from 3 to $12 \mathrm{~dB}$ and the corresponding capacities over this SNR range, for different antenna configurations are plotted in Fig. 2. The number of elements on the transmit side and the receive side is taken to be three. For the printed dipole array $\varepsilon_{\mathrm{r}}=2.55, \mathrm{a}=\mathrm{b}=0.5 \lambda_{0}, \mathrm{~L}$ $=0.39 \lambda_{0}$ and $\mathrm{W}=0.01 \lambda_{0}$. Fig. 3 shows the mean capacity as a function of SNR for $\mathrm{H}$ and $\mathrm{V}$ shaped antenna configurations.

Figure 4 shows the comparison of exact analytical, high SNR analytical, results for Ergodic capacity of AF MIMO dual-hop systems with different antenna configurations.

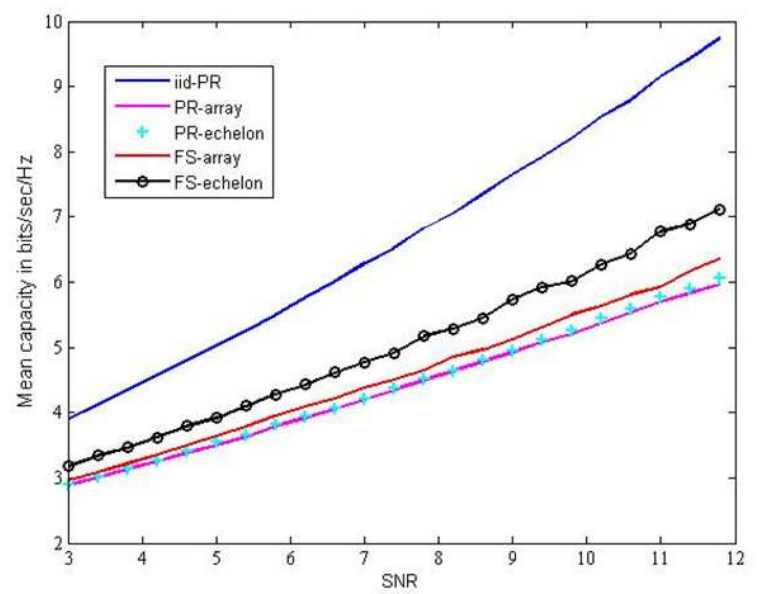

Fig. 2: Mean Capacity versus $\mathrm{SNR}$ in $\mathrm{dB}$ for 3 element Printed dipole (PR) and Freestanding dipole (FS) arrays with side by side and echelon arrangements.
In Fig. 5 high SNR power offset shift $\delta(\mathrm{nd}, \mathrm{k})$ is plotted against nd, for $\mathrm{k}=1, \mathrm{k}=2$ and $\mathrm{k}=4$. As expected, for a fixed value of $\mathrm{k}, \delta$ (nd, $\mathrm{k}$ ) is an increasing function of nd, approaching a limit of $0 \mathrm{~dB}$ as nd $\rightarrow \infty$. Figure. 6 compares the closed-form upper bound with the exact analytical Ergodic capacity based on for two different AF MIMO dual-hop system configurations. The results are shown as a function of SNR $\rho$, with $\alpha=2 \rho$. We see that the closed-form upper bound is very tight for all SNRs, for both system configurations considered. Figure 7 shows the comparison between the Conventional point-Point MIMO System and the DualHop AF MIMO Relay System.

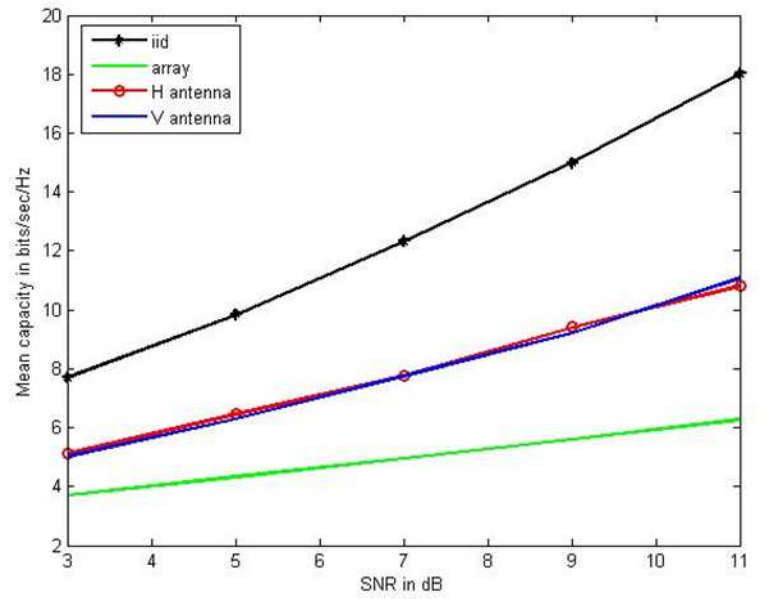

Fig. 3: Mean capacity as a function of SNR for $\mathrm{H}$ and $\mathrm{V}$ shaped antenna configurations

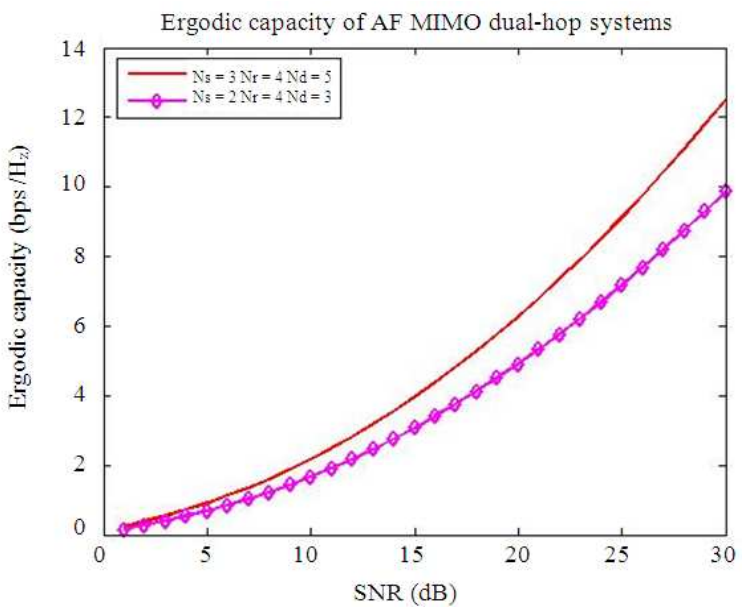

Fig. 4: Comparison of exact analytical, high SNR analytical, results for Ergodic capacity of AF MIMO dual-hop systems with different antenna configurations 


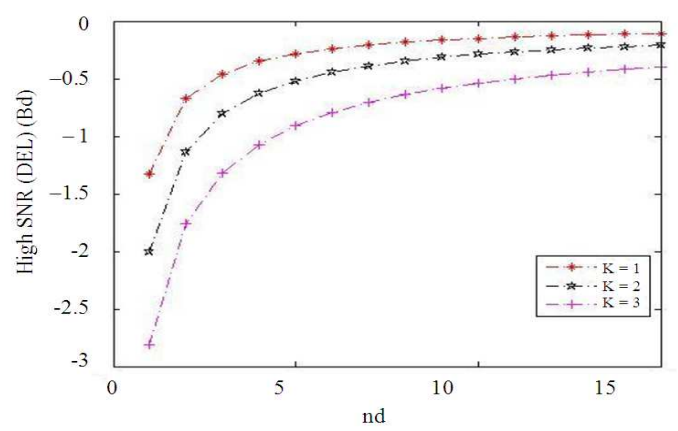

Fig. 5: High SNR in decibels, obtaining by adding either (a) one antenna to the destination, (b) two antennas to the destination, or (c) four antennas to the destination. Results are shown for $\mathrm{ns}=\mathrm{nr}=1$ and $\alpha / \rho=2$

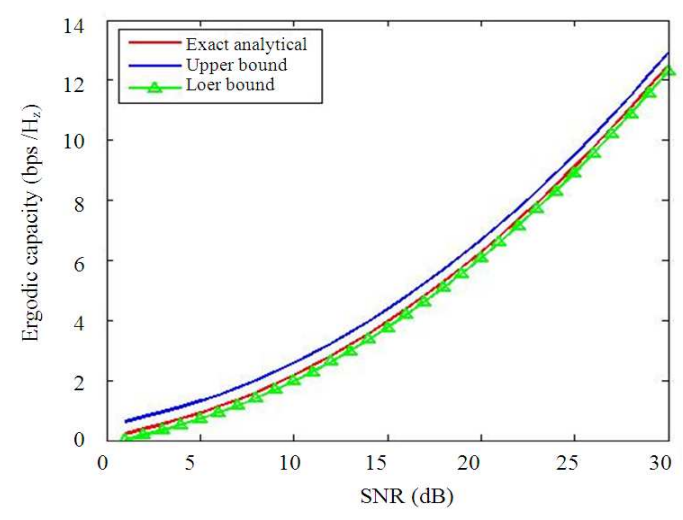

Fig. 6: Ergodic capacity of AF MIMO dual-hop systems with different antenna configurations for exact analytical, high SNR analytical and Monte Carlo simulation results are shown for $\alpha / \rho=2$

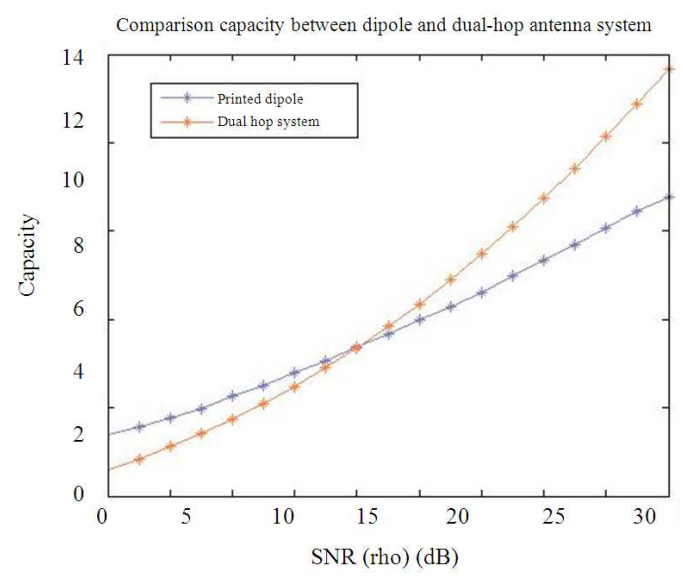

Fig. 7: Capacity of dual-hop AF MIMO relay system and conventional point-point MIMO system.

\section{DISCUSSION}

In Fig. 2, the echelon dipole configuration performs well compared to side by side configurations. This is possibly due to the fact that the echelon configuration has very little mutual coupling. Only the array configuration of the mobile station varied in the simulation. In the receiver side, the side by side or echelon configuration is kept with a spacing of $2 \lambda$. One hundred channel realizations were taken for the simulations. The separation between mobile stations is held constant $0.1 \lambda$. In Fig. 3, H-type, V -type dipole array configurations show less mutual impedance and dissimilar radiation patterns than side by side configuration and hence they can offer better capacity than side by side case. The number of elements on the transmit side and the receive side is taken to be six. The slanting angle of the ' $\mathrm{V}$ ' shaped array is $45^{\circ}$. Since the ' $\mathrm{V}$ ' shaped and ' $\mathrm{H}$ ' shaped are dual polarized, the capacity due to them will be larger than side by side case. The side by side case with spacing between the elements $0.2 \lambda$ is also given for comparison. The Fig. 4 shows the capacity analysis of AF MIMO dual-hop system with different array configuration. The results are taken upon the condition of $\alpha / \rho=2$. The result clearly indicates that when number of source and destination antenna is increased, keeping the number of relay antenna as same, the Ergodic capacity is increased. The Capacity of Dual-Hop relay system is less than the Conventional point-Point MIMO System when the SNR value is less than $10 \mathrm{~dB}$. But for high SNR value (SNR value greater than $10 \mathrm{~dB}$ ) the Capacity value is greater than the Conventional point-Point MIMO System.

\section{CONCLUSION}

In this study the MIMO capacities of echelon, Hshaped and V -shaped dipole array and printed dipole antenna configurations are evaluated using Monte Carlo simulations and compared with the usual side by side configuration. The echelon, $\mathrm{H}-$ shaped and V - shaped dipole array configurations show less mutual impedance and dissimilar radiation patterns than side by side configuration and hence they can offer better capacity. This study has presented an analytical characterization of the Ergodic capacity of AF MIMO dual-hop relay channels under the common assumption that CSI is available at the destination terminal, but not at the relay or the source terminal. We analysed the 
Ergodic capacity, for the high SNR regime. Simplified closed-form upper and lower bounds were also presented, which were shown to be tight for all SNRs. A comparative relationship between the conventional point-point MIMO system and dual hop AF MIMO relay network is also studied. The analytical results were validated through comparison with numerical simulations.

\section{REFERENCES}

Sayadi, F., M. Ismail, N. Misran, K. Jumari and M. Abdullah 2009. Efficient detection algorithm for a multiple-input and multiple-output multiuser multicarrier code division multiple access in timevarying channels. Am. J. Eng. Applied Sci., 2: 635642. DOI: $10.3844 /$ ajeassp.2009.635.642

Gesbert, D., 2003. From theory to practice: An overview of MIMO space-time coded wireless systems. IEEE J. Select. Areas Commun., 21: 281302. DOI: 10.1109/JSAC.2003.809458

Poongodi, C., K. Dineshkumar, D. Deenadhayalan and A. Shanmugam, 2010. An algorithm for reconfigurable communication system based on MIMO/adaptive antennas and their performance analysis. Proceedings of the IEEE International Conference on Communication and Computational Intelligence, Dec. 27-29, IEEE Xplore Press, Erode, pp: 269-274.

Svantesson, T. and A. Ranheim, 2001. Mutual coupling effects on the capacity of multielement antenna systems. Proceedings of the IEEE International Conference Acoustics, Speach, Signal Processing, May 7-11, IEEE Xplore Press, Salt Lake City, UT, USA., $\quad$ pp: 2485-2488. DOI: 10.1109/ICASSP.2001.940505

Balanis, C.A., 1997. Antenna Theory: Analysis and Design. 2nd Edn., Wiley, New York, ISBN: 0471592684, pp: 941.

Wagner, J., B. Rankov and A. Wittneben, 2007. On the asymptotic capacity of the Rayleigh fading amplify-and-forward MIMO relay channel. Proceedings of the IEEE International Symposium. on Information Theory, June 24-29, IEEE Xplore Press, Nice, pp: 2711-2715. DOI: 10.1109/ISIT.2007.4557628

Lee, K.J., J.S. Kim, G. Caire and I. Lee, 2010. Asymptotic Ergodic capacity analysis for MIMO amplify-and-forward relay networks. IEEE Trans. Wireless Commun, 9: 2712-1217. DOI: 10.1109/TWC.2010.071410.091701
Morgenshtern, V.I. and H. Bolcskei, 2006. Random matrix analysis of large relay networks. Proceedings of the 44th Allerton Conference Communication, Sept. 27-29, Allerton House, UIUC, Illinois, USA., pp: 106-112.

Svantesson, T., 2001. A physical MIMO radio channel model for multi-element multi-polarized antenna systems. Proceedings of the IEEE 54th Vehicular Technology Conference, Oct. 7-11, IEEE Xplore Press, Atlantic City, NJ, USA., pp: 1083-1087. DOI: 10.1109/VTC.2001.956941

Paulraj, A., R. Nabar and D.Gore, 2003. Introduction to Space-Time Wireless Communications. 1st Edn., Cambridge University Press, Cambridge, ISBN: 0521826152, pp: 277.

Carter, P.S., 1932. Circuit relations in radiating systems and applications to antenna problems. Proc. Inst. Radio Eng., 20: 1004-1041. DOI: 10.1109/JRPROC.1932.227723

Pozar, D., 1985. Analysis of finite phased arrays of printed dipoles. IEEE Trans. Antennas Propagat., 33: 1045-1053. DOI: 10.1109/TAP.1985.1143501

Pozar, D., 1982. Input impedance and mutual coupling of rectangular microstrip antennas. IEEE Trans. Antennas Propagat., 30: 1191-1196. DOI: 10.1109/TAP.1982.1142934

Garg, R., 2001. Microstrip Antenna Design Handbook. 1st Edn., Artech House, Boston, Mass., ISBN: 0890065136, pp: 845.

Pozar, D.M., 1983. Improved computational efficiency for the moment method solution of printed dipoles and patches. Electromagnetic., 3: 299-309. DOI: $10.1080 / 02726348308915190$

Morgenshtern, V.I. and H. Bolcskei, 2007. Crystallization in large wireless networks. IEEE Trans. Inform. Theory, 53: 3319-3349. DOI: 10.1109/TIT.2007.904789

Wang, J., X. Sha, L. Sun and Z. Zhang, 2009. Performance study of cooperative diversity system over nakagami-m fading channels. Inform. Technol. J., 8: 1013-1019. 